\title{
The extracellular form of NAMPT contributes to creating a proinflammatory environment in chronic lymphocytic leukemia
}

\section{Running title: The role of NAMPT in CLL}

Valentina Audrito ${ }^{1,2}$, Sara Serra ${ }^{1,2}$, Tiziana Vaisitti ${ }^{1,2}$, Nadia Raffaelli ${ }^{3}$, Luca Laurenti ${ }^{4}$, Giovanni D'Arena ${ }^{5}$, Davide Rossi ${ }^{6}$, Gianluca Gaidano ${ }^{6}$, Menico Rizzi ${ }^{7}$ and Silvia Deaglio ${ }^{1,2}$

${ }^{1}$ Immunogenetics Unit, Dept. of Medical Sciences, University of Torino, Italy; ${ }^{2}$ Human Genetics Foundation

(HuGeF), Torino, Italy; ${ }^{3}$ Dept. of Molecular Pathology and Innovative Therapies, Section of Biochemistry, Università Politecnica delle Marche, Ancona, Italy; ${ }^{4}$ Hematology Chair, Catholic University of the Sacred Heart, Rome, Italy; ${ }^{5}$ Centro di Riferimento Oncologico della Basilicata (CROB), Rionero in Vulture, Italy; ${ }^{6}$ Division of Hematology, Dept. of Translational Medicine, "Amedeo Avogadro" University of Eastern Piedmont and AOU Maggiore della Carità, Novara, Italy; ${ }^{7}$ Dept. of Chemical, Food, Pharmaceutical and Pharmacological Sciences, "Amedeo Avogadro" University of Eastern Piedmont, Novara, Italy.

E-mail contact: valentina.audrito@unito.it

Malignant cells are characterized by a higher NAD turnover rate than normal cells, making this biosynthetic pathway an attractive target for cancer treatment. Increasing evidence indicate that NAD plays important roles not only in energy metabolism, but also in calcium homeostasis and inflammation. Here we investigated the biologic significance of a rate-limiting enzyme of the NAD synthesis pathway, nicotinamide phosphoribosyl transferase (NAMPT), focusing our attention on the extracellular form (eNAMPT), which exerts cytokine/adipokine-like actions in different tumor models as well as in acute and chronic inflammatory-metabolic diseases. The role of eNAMPT and its mechanisms of action have been addressed in chronic lymphocytic leukemia (CLL), an indolent lymphoproliferative disorder, strongly dependent on a growth supportive environment and on nucleotide/nucleoside metabolism. The aim of this work is to test whether eNAMPT plays a role in shaping the leukemic environment, by generating proinflammatory conditions that favor tumor expansion.

Results indicated that: i) B-CLL lymphocytes expressed higher levels of NAMPT mRNA compared to normal B lymphocytes obtained from age- and sex-matched donors; ii) plasma levels of eNAMPT were also significantly higher in CLL patients $(n=80)$ compared to controls $(n=20)$; and iii) activation of purified CLL cells was followed by eNAMPT secretion, indicating that it is the leukemic component that actively releases eNAMPT. Then we addressed the question whether this cytokine may play an active role in the leukemic microenvironment. Treatment of PBMCs, but not B purified CLL lymphocytes, for 5 days with recombinant NAMPT resulted in an increased numbers of adherent cells $\left(\mathrm{CD} 11 \mathrm{~b}^{+}\right)$, displaying intracellular vacuoles and 
granules, consistent with macrophage differentiation, and in significant induction and secretion of proinflammatory cytokines. Furthermore, long-term exposure to eNAMPT enhanced the formation and the phagocytosis ability of nurse-like cells (NCLs), a $\mathrm{CD} 68^{+}$myeloid population and an essential component of the CLL microenvironment. Moreover, treatment of NLCs with eNAMPT triggered rapid phosphorylation of Erk1/2, STAT3 and nuclear translocation of the NF-kB component p65. Lastly, preliminary data indicated that NAMPT enzymatic activity is not required for its extracellular functions, as highlighted by the inability of i) nicotinamide mononucleotide (NMN), a product of the NAMPT activity, to induce STAT3 phosphorylation and cytokines secretion, as well as ii) NAMPT inhibitor FK866 to block eNAMPTdependent STAT3 activation.

Taken together, these data support the hypothesis that eNAMPT, secreted by CLL cells, contributes to the formation of a proinflammatory environment, driving recruitment and differentiation of myeloid populations, that provides anti-apoptotic and pro-survival signals to leukemic cells. 